\title{
Deeper look at student learning of quantum mechanics: The case of tunneling
}

\author{
S. B. McKagan, ${ }^{1}$ K. K. Perkins, ${ }^{2}$ and C. E. Wieman ${ }^{1,2,3}$ \\ ${ }^{1}$ JILA, University of Colorado and NIST, Boulder, Colorado 80309, USA \\ ${ }^{2}$ Department of Physics, University of Colorado, Boulder, Colorado 80309, USA \\ ${ }^{3}$ Department of Physics, University of British Columbia, Vancouver, British Columbia, Canada V6T 1Z1
}

(Received 19 March 2008; published 8 October 2008)

\begin{abstract}
We report on a large-scale study of student learning of quantum tunneling in four traditional and four transformed modern physics courses. In the transformed courses, which were designed to address student difficulties found in previous research, students still struggle with many of the same issues found in other courses. However, the reasons for these difficulties are more subtle, and many new issues are brought to the surface. By explicitly addressing how to build models of wave functions and energy and how to relate these models to real physical systems, we have opened up a floodgate of deep and difficult questions as students struggle to make sense of these models. We conclude that the difficulties found in previous research are the tip of the iceberg, and the real issue at the heart of student difficulties in learning quantum tunneling is the struggle to build the complex models that are implicit in experts' understanding but often not explicitly addressed in instruction.
\end{abstract}

DOI: 10.1103/PhysRevSTPER.4.020103

PACS number(s): 01.40.Fk, 01.50.ht, 03.65.Xp

\section{INTRODUCTION}

Tunneling is a surprising result that has served to validate the theory of quantum mechanics by explaining many realworld phenomena such as alpha decay, molecular bonding, and field emission and has resulted in applications such as scanning tunneling microscopes. As a case study in the counterintuitive yet applicable nature of quantum mechanics, tunneling is an important part of any introductory course in modern physics or quantum mechanics.

An examination of modern physics and quantum mechanics textbooks, course syllabi, and interviews with faculty who have taught such courses suggest that instruction in tunneling should help students achieve the following learning goals: (1) calculate or discuss qualitatively (depending on the level of the course) the probability of tunneling for various physical situations, (2) describe the meaning of the potentialenergy and wave-function graphs, (3) visualize how these graphs would change if the physical situation were altered (e.g., changing barrier height and width), and (4) relate the mathematical formalism and graphical representation of tunneling to the phenomenon of tunneling in the real world.

Tunneling has been a favorite topic of physics education researchers specializing in quantum mechanics, who have found that many students have a great deal of trouble understanding even the most basic aspects of this topic. ${ }^{1-7}$ In designing a transformed course in modern physics for engineering majors, ${ }^{8}$ we drew on the literature of previous research to develop a curriculum aimed at addressing known student difficulties in understanding quantum tunneling. ${ }^{9}$ Throughout the process of developing and refining this course, we carried out a study to answer the following research questions:

(1) Does our transformed curriculum help to address common student difficulties in learning tunneling?

(2) Are our students achieving the learning goals described above?

(3) What are the practices that support or hinder the achievement of these goals?
We find that our curriculum does help students overcome common difficulties and achieve our learning goals. While the common difficulties reported in the literature do arise in the transformed classes, they are less prevalent than in comparable traditional classes, and they often arise for different reasons than discussed in the previous literature. Further, we find new difficulties that have not been previously reported, associated with a struggle to make sense of the models of quantum mechanics and relate them to the real world.

The difficulties discussed in the literature are associated with the inability to apply the quantum model to abstract model systems such as square barriers and square wells. Our transformed course focuses on relating these abstract model systems to reality, and our research shows that the difficulties discussed in the literature are surface features, masking a much more serious problem: In tunneling, as in other aspects of quantum mechanics, students fail to grasp the basic models that we are using to describe the world as anything more than abstract model systems. These models include wave functions as descriptions of physical objects, potentialenergy graphs as descriptions of the interactions of those objects with their environments, and total energy as a delocalized property of an entire wave function that is a function of position. Thus, even when students can successfully overcome problems that previous research has elucidated, such as relating wave functions to potentials, they may not know what a wave function or a potential is.

Hestenes ${ }^{10}$ pointed out that while "a physicist possesses a battery of abstract models with ramifications already worked out or easily generated," standard physics instruction often treats these models implicitly rather than explicitly. While this is true even in introductory physics, the problem is more serious in quantum mechanics, where the models are particularly abstract, and the connection between the models and the real world is more tenuous. Standard instruction in quantum mechanics, including tunneling, does not provide students with enough information to make sense of these models, to relate them to anything real, or even to recognize that they exist. We have achieved a degree of success in teaching 


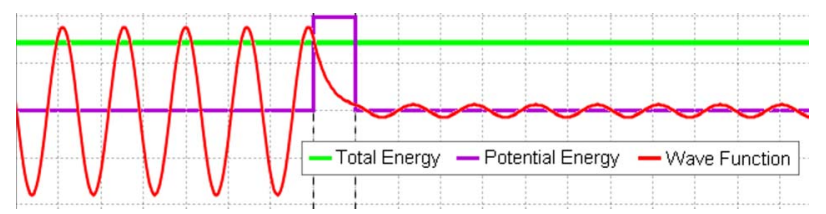

FIG. 1. (Color) The standard presentation of quantum tunneling: a plane wave tunneling through a square potential barrier. Total energy, potential energy, and the real part of the wave function are all drawn on the same graph, and the real part of the wave function is labeled as simply wave function.

quantum tunneling by making these models more explicit and connecting them to real-world applications and suggest further changes in this direction.

\section{STANDARD PRESENTATION OF QUANTUM TUNNELING}

Most textbooks on modern physics and quantum mechanics have a discussion of quantum tunneling. The discussion is remarkably similar throughout these books, with the main difference being that modern physics textbooks give less detail. Tunneling is defined as a wave function passing through a potential-energy barrier that is greater than its total energy. The typical presentation includes an analysis of the planewave solution to the Schrödinger equation for a square potential-energy barrier, as shown in Fig. 1. Often the wave function, potential energy, and total energy are drawn on the same graph, a practice which research has shown to lead to student confusion ${ }^{3,6}$ and which thoughtful authors have avoided since the 1970s. ${ }^{11}$ Depending on the level of the textbook, the reflection and transmission coefficients are either derived or given. This is typically followed by a discussion of some applications of quantum tunneling, such as alpha decay, scanning tunneling microscopes, and the inversion of ammonia molecules. Some textbooks also include a discussion of tunneling wave packets, occasionally showing pictures of a tunneling wave packet taken from a numerical simulation such as in Ref. 12. Wave packets and applications are nearly always relegated to the end of the discussion of tunneling.

In examining the standard presentation of tunneling, one may ask how it aligns with the learning goals in Sec. I. The standard presentation certainly gives students practice in calculating relevant quantities for the case of a plane wave and square barrier, but it does not give students the tools to extend these calculations to more realistic systems. It also includes both a mathematical model and a discussion of physical applications of this model. However, we argue that it does not provide sufficient links between the two. For example, there is almost never a discussion of what physical system could produce the square barrier shown in Fig. 1 or of how a plane wave relates to a real particle. Further, when real applications are discussed, their potential-energy graphs are often not discussed, making it harder for students to relate the applications to the mathematical model. Thus, the standard presentation does not provide students with the tools to extend the model of tunneling beyond square barriers to the more complicated potentials involved in real physical systems, either quantitatively or qualitatively.

\section{PREVIOUS PHYSICS EDUCATION RESEARCH ON QUANTUM TUNNELING}

Many researchers have documented student difficulties in learning quantum tunneling. ${ }^{1-7}$ These researchers, working at many institutions in the United States and Sweden, have found a fairly consistent list of student difficulties.

The most common difficulty, discussed in all these references, is the belief that energy is lost in tunneling. The correct description of energy in quantum tunneling is that because there is no dissipation in the Schrödinger equation, energy is conserved, as can be seen in Fig. 1, where the total energy is constant throughout. The barrier itself represents the potential energy, which is zero on the left and right and some positive constant inside the barrier. ${ }^{13}$ The kinetic energy is equal to the total energy on the left and right and is negative inside the barrier. Ambrose ${ }^{1}$ and $\mathrm{Bao}^{2}$ reported the student belief that kinetic energy is lost in tunneling, although later research shows that this difficulty is not limited to kinetic energy: Morgan et al. ${ }^{3}$ quoted students as saying that "energy" is lost, without specifying which kind of energy, and in our own work, we found that most students who thought that energy is lost did not have a clear idea of which energy is lost. When asked, they were just as likely to say potential, kinetic, or total energy and often used two or even all three types of energy interchangeably within the same explanation. $^{7}$

There are two common explanations in the literature for the belief that energy is lost in tunneling. The first explanation (reason 1), attributable to the fact that most textbooks and lecturers draw the energy and the wave function on the same graph, is that students confuse the two, believing that the energy, like the wave function, decays exponentially during tunneling. ${ }^{2,3,6}$ This explanation is reminiscent of the classic confusion between velocity and acceleration in introductory physics, ${ }^{14-19}$ while students can correctly recite definitions and formulas for wave function and energy, they fail to distinguish between the two when solving problems. The second explanation (reason 2) is that students think that " "work' is done on or by the particles while inside the potential barrier" or that energy is "dissipated" as in a physical macroscopic tunnel. ${ }^{3}$ Many researchers report on student interviews showing that both these explanations are common among students. ${ }^{1-7}$

A third possible explanation (reason 3 ) suggested by $\mathrm{Bao}^{2}$ is that students may be thinking of mechanical or electromagnetic waves, in which the energy of the wave is related to the amplitude. However, no evidence is presented to support this explanation of student thinking. In our observations and interviews in traditional modern physics courses, few students have sufficient understanding of mechanical or electromagnetic waves to cause problems in their interpretation of the amplitude of matter waves, and none have used such an explanation. As discussed in Sec. VI B, we do see some evidence of students using this explanation for energy loss in our transformed modern physics course, in which the depen- 
(a)

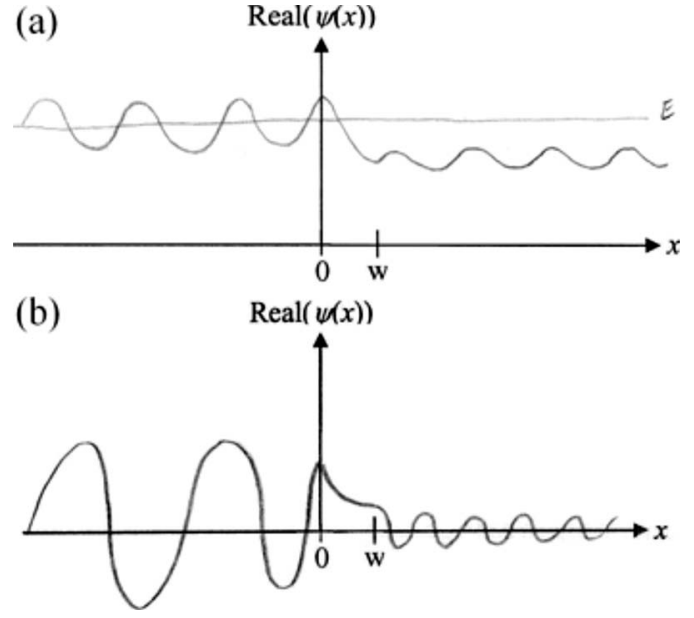

FIG. 2. Common student difficulties reported in the literature: incorrectly drawing the real part of the wave function with (a) an offset between the horizontal axes on the left and right sides of the barrier and (b) a smaller wavelength on the right than on the left. These drawings are taken from student responses to an exam question asking students to draw the real part of the wave function, as discussed in Sec. VI. We have observed physics faculty making drawings similar to both (a) and (b), and a popular introductory quantum mechanics textbook contains a figure similar to (b). ${ }^{42}$

dence of amplitude on energy in electromagnetic waves is heavily stressed.

Other common student difficulties reported in the literature are the belief that reflection at a barrier is due to particles having a range of energies, ${ }^{1}$ incorrectly drawing the wave function with an offset between the horizontal axes of the wave function on the left and right sides of the barrier, as in Fig. 2(a), ${ }^{3}$ incorrectly drawing the wave function with a smaller wavelength on the right than on the left, as in Fig. $2(b),{ }^{1,3}$ and misinterpreting the meaning of the wavelength and amplitude of the wave function.

In addition to these common student difficulties, in our own previous research we found that many students do not know what the potential-energy graph represents. ${ }^{7}$ Our results from student interviews are supported by many conversations with practicing physicists who report having successfully completed quantum mechanics courses as students without realizing what a potential well was until much later. We believe that this problem is due to the lack of physical context for potential-energy graphs in the standard treatment discussed in Sec. II. We will return to this issue later.

Brookes and Etkina ${ }^{20,21}$ argued that physicists talk about potential using a metaphor of a physical object, as illustrated by the terms "potential well," "potential barrier," and "potential step." Because these metaphors are implicit and their limitations are not discussed, students have a tendency to overextend them, leading to many of the student difficulties that other researchers have documented. This analysis ${ }^{20}$ overlaps with ours in that they also pointed out that physics professors are not explicit in discussing the limitations of models.

\section{IMPROVED CURRICULUM FOR TEACHING QUANTUM TUNNELING}

As part of the transformation of a modern physics course for engineering majors, ${ }^{8}$ we developed a curriculum for teaching quantum tunneling. The course design was based on physics education research (PER) using interactive engagement techniques such as peer instruction and collaborative homework sessions, focusing on real-world applications, and addressing common student difficulties. The curriculum on quantum tunneling was designed to address common student difficulties with this topic, which were known from previous research (see Sec. III). Throughout the course, we emphasized building models and relating them to the real world, asking students in lecture, homework, and exams both to construct their own models and to explain models that had been presented to them. ${ }^{9}$

\section{A. Addressing student difficulties with energy loss}

Several aspects of the instruction were designed to address the belief that energy is lost in tunneling. As discussed in Sec. III, previous research cites two reasons that students believe energy is lost in tunneling: (1) treating energy and wave function interchangeably and (2) invoking dissipation.

To address reason 1, we were careful to draw energy and wave function on separate graphs. However, since the representation in Fig. 1, in which they are plotted on the same graph, is ubiquitous in textbooks and other literature, it is impossible to avoid students being exposed to it. This representation has been so ingrained in us by our own education that we had to be on guard to keep from drawing graphs this way ourselves. Therefore, we also used concept questions (multiple choice questions posed in class that students discuss in small groups and answer using a personal response system) and homework questions to elicit student confusion between energy and wave function and address it directly. Figure 3 shows an example of a concept question used to address this confusion.

To address reason 2, we emphasized energy conservation and the lack of dissipation in the Schrödinger equation. One key feature of our curriculum was a tutorial ${ }^{9}$ adapted from the Quantum Tunneling Tutorial in the Activity-Based Tutorials Volume 2, developed by Wittmann et al. ${ }^{22}$ This tutorial was designed to address the belief that energy is lost in tunneling by asking students to work out the total, kinetic, and potential energies in each region and answer questions about energy conservation.

\section{B. Giving potential energy a physical context}

We also designed our curriculum to address our previous finding that students are often confused by the meaning of the potential-energy function. ${ }^{7}$ We consistently gave a physical context for potential-energy functions, presenting square wells and barriers as illustrations of real physical systems, rather than mere abstractions. It is worth noting that it was a great challenge for our team of three expert physicists, including one Nobel Laureate, to think of even a single real physical system represented by a square well or a square 


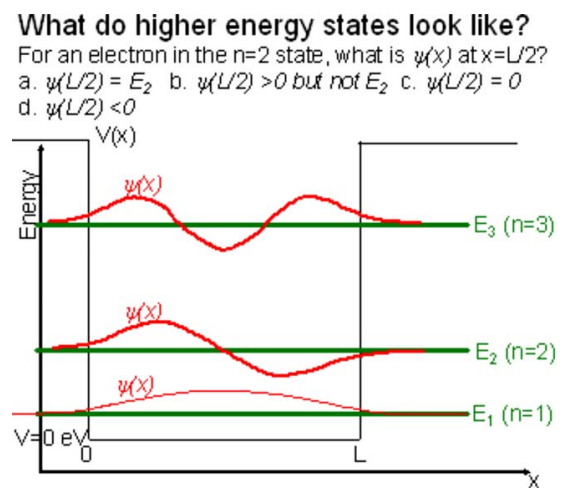

FIG. 3. (Color) A concept question designed to elicit student confusion between energy and wave function. The correct answer is $\mathrm{C}$ but students who do not understand the meaning of superimposing a wave-function graph on an energy graph may be inclined to answer A. When we ask this question in class, it generates a large amount of discussion. While most students $(73 \%-88 \%$, depending on the semester) eventually answer the question correctly, listening in on student discussions reveals that most do not know the answer right away and only figure it out through vigorous debate with their neighbors. Even after discussion, 9\%-19\% give answer A.

barrier. This illustrates that for content that is outside of our area of research, even physicists sometimes do not know how an idealized textbook model can be applied to the real world.

The physical examples that we decided to use in our course are illustrated in Fig. 4: an electron in a short wire as the context for a square well and an electron traveling through a long wire with a thin air gap as the context for a square barrier. Because the electrons are free to move around within the wire, the potential energy of an electron is constant anywhere inside the wire (and we can arbitrarily set the constant value to zero). Because the electrons are bound to the wire and require energy to escape, their potential energy outside the wire will be a larger constant, so that the potential energy of the system is well approximated by a finite square well. In lecture, we ask students to predict the value of the potential energy outside the wire by reminding them of the

(a)
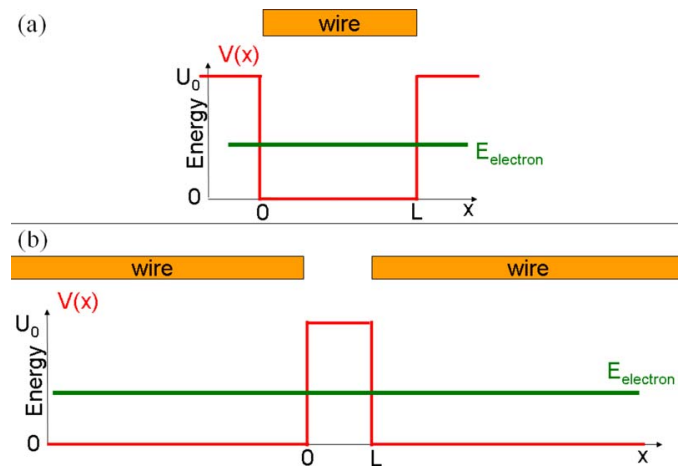

FIG. 4. (Color) Physical contexts for (a) a square well and (b) a square barrier. A square well with width $L$ and height $U_{0}$ represents a wire with length $L$ and work function $U_{0}$. A square barrier with width $L$ and height $U_{0}$ represents two long wires with work function $U_{0}$ separated by an air gap with length $L$. energy required to kick an electron out of a metal in the photoelectric effect, which they learned about earlier in the course. Students discuss this question in small groups and most eventually recognize that the potential energy outside the wire will be given by the work function of the metal. After the first semester, we also explained the shape of the potential energy of a wire by providing a microscopic model in which you add up all the Coulomb potentials of the ion cores of the atoms in the wire, as illustrated in Fig. 10.

We chose the physical context of an electron in a wire because it has practical applications for real circuits. While there are a few textbooks that provide physical examples of tunneling (an electron bouncing back and forth between two capacitors with tiny holes in them for a square well ${ }^{23}$ and an electron traveling through a series of metal tubes held at different voltages for a square barrier ${ }^{24}$ ), these examples are so artificial that no one would ever create such a system for any reason other than to demonstrate the abstract potentials used in introductory quantum mechanics courses. We decided against using the example of a charged bead moving along a wire held at different potentials that was used in the original version of the Activity-Based Tutorials ${ }^{22}$ also because it seemed excessively artificial.

Our curriculum included many opportunities for students to practice building models of how potential-energy graphs relate to physical systems. For example, in interactive lectures, homework problems, and a tutorial we asked students to build up potential-energy diagrams for systems such as an electron in a wire, a scanning tunneling microscope, and a nucleus undergoing alpha decay. We also asked students to reason through the physical meaning of the potential energy for various systems.

Further, we used the term "potential energy," rather than the shorthand "potential," to avoid confusion. ${ }^{25}$ Although it would be preferable to use the symbol $U$, rather than the common convention $V$, for potential energy, to help students relate the potential energy in quantum mechanics to the potential energy in other areas of physics, we used $V$ in order to be consistent with the textbook we chose for the first semester. However, we repeatedly emphasized the meaning of this symbol and explicitly pointed out the inconsistency in notation among different areas of physics.

\section{Quantum Tunneling simulation}

The standard presentation of quantum tunneling discussed in Sec. II provides an abstract and decontextualized model that is difficult to visualize or connect to reality. The content of this presentation is artificially constrained by what can be calculated. Students learn to calculate transmission coefficients for plane waves tunneling through square barriers not because this is a relevant problem but because this is the only tunneling problem that can reasonably be calculated analytically. With modern computational techniques, however, it is no longer necessary for the curriculum to be so constrained.

We designed the Quantum Tunneling and Wave Packets simulation $^{26}$ (see Fig. 5) to provide easily accessible interactive visual models of tunneling of wave packets and plane waves in a variety of physical situations, thus removing many constraints on curriculum. With the simulation, we can 


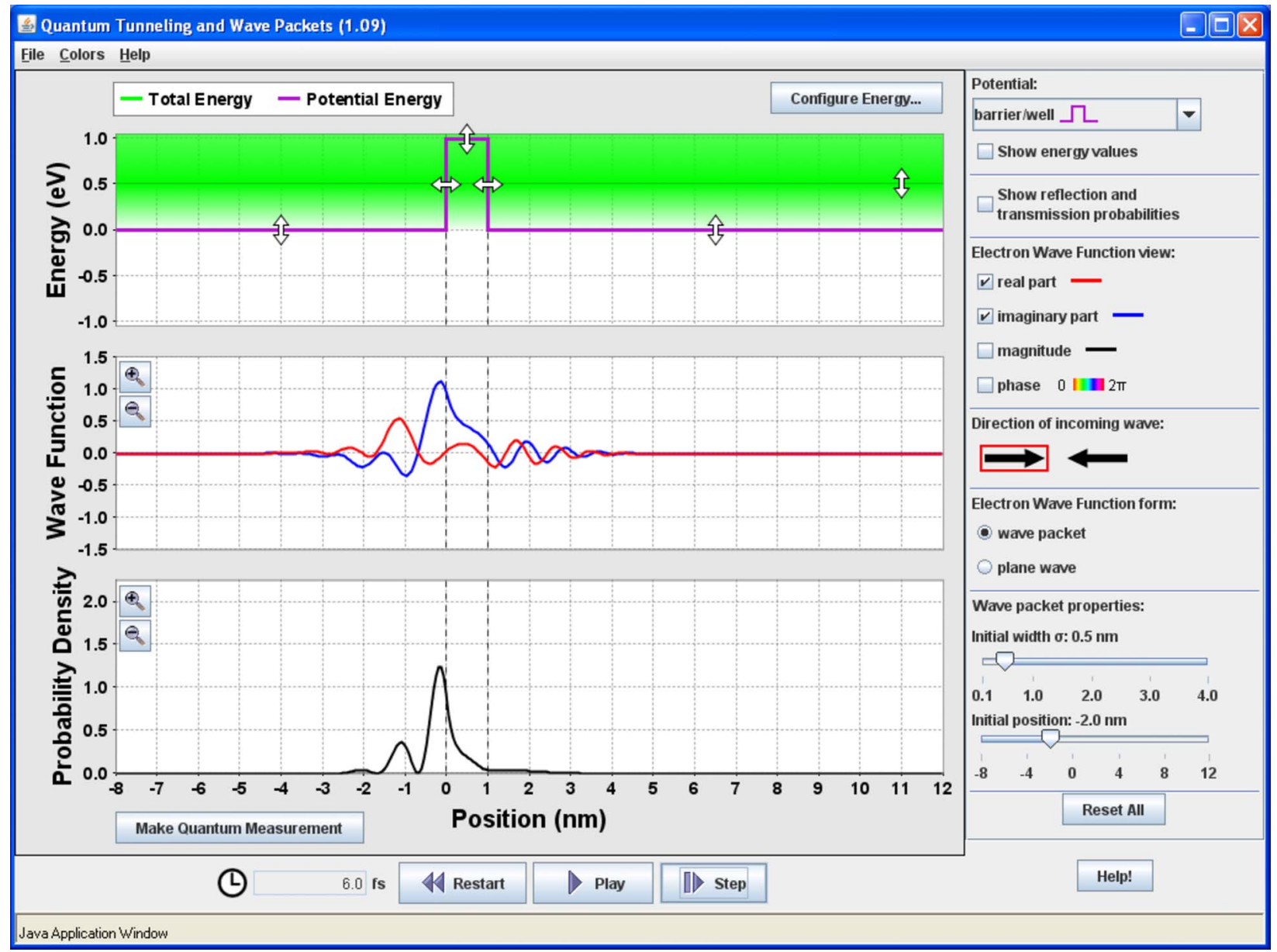

FIG. 5. (Color) The Quantum Tunneling and Wave Packets simulation provides interactive visual models of tunneling of wave packets and plane waves in a variety of physical situations and removes constraints imposed on curriculum by what problems can be easily calculated.

begin our instruction with wave packets, rather than plane waves, so that students can develop a visual model of what is happening in time and space in quantum tunneling. This simulation was developed as part of the Physics Education Technology (PhET) project, ${ }^{27}$ which provides free interactive computer simulations for teaching physics. Like other PhET simulations, the Quantum Tunneling and Wave Packets simulation is highly interactive, allowing students to change the potential and total energies by dragging on the graph, so that they can quickly explore a wide variety of physical situations that would be cumbersome to calculate. The simulation also provides a wide variety of representations, allowing students to view the real part, imaginary part, magnitude, and phase of the wave function. To address the problem of students treating energy and wave function interchangeably, these quantities are displayed on separate graphs in the simulation.

We note that in the first semester of the reformed course, before developing the simulation, we attempted to use existing simulations on quantum tunneling, as many have already been developed by others. ${ }^{12,28-33}$ However, we found that students quickly became frustrated by the limitations of these simulations. For example, students wanted to be able to adjust the properties of the wave packet and/or barrier and to see the real part of the wave function rather than just the magnitude. Further, all these simulations had features that research has demonstrated to be ineffective for student learning, such as plotting the wave function and the potential on the same graph, ${ }^{3,6}$ using a phase color representation ${ }^{34}$ and limited interactivity. ${ }^{35}$ As a result, we designed our own simulation that we used in the course starting in Spring 2006.

\section{STUDY}

In order to answer the research questions in Sec. I, we collected qualitative and quantitative data on student thinking about quantum tunneling in eight modern physics courses over a five-semester period. Five of these courses were for engineering majors and three were for physics majors. Four of the engineering major courses were taught using the transformed curriculum described in Sec. IV. The first two semesters of the transformed course were taught by the authors and the next two by another professor in the PER group. ${ }^{36}$ The remaining courses in the study were taught in a traditional manner along the lines of Sec. II, with minimal or no use of peer instruction, collaborative homework sessions, focusing on real-world applications, addressing common student difficulties, or interactive simulations. Some of these courses used clickers, but their use was less frequent and 
involved less discussion than in the transformed courses.

The qualitative data we collected consist of observations of students in lectures and problem-solving sessions, student responses to essay questions on homework and exams, and student interviews. Interviews included students participating in a case study project, interviews on the Quantum Mechanics Conceptual Survey (QMCS), ${ }^{37,38}$ which includes questions on tunneling, ${ }^{7}$ and interviews on the Quantum Tunneling and Wave Packets simulation described in Sec. IV C. The quantitative data consist of student responses on the QMCS, homework, and exams.

Observations included approximately 200 lectures (20 on tunneling) and 50 problem-solving sessions (5 on tunneling). In lectures, a researcher (S.B.M.) took detailed field notes during and after class, writing down all questions that students asked of the lecturer and summarizing student discussions during clicker questions. The researcher also took field notes immediately after problem-solving sessions, writing summaries of her interactions with and observations of students working on homework. Undergraduate learning assistants (LAs) who were hired to facilitate student discussion during lecture and problem-solving sessions also took field notes, which provided an additional perspective.

The case study interviews involved six students who were taking the transformed course in Spring 2006. Each of these students participated in 11 interviews throughout the semester. Two of the interviews with each of these students (total of 12 interviews) focused on quantum tunneling. In the first interview on tunneling, students were asked to go over the tunneling tutorial that they had already completed in class and homework. In the second interview, they were asked a series of questions about what happens to the energy and probability of tunneling for an electron approaching a barrier when the height or width of the barrier is changed, culminating in the question in Fig. 7.

We conducted interviews on the QMCS with 47 students, including 24 from transformed courses and 23 from traditional courses. In these interviews, most of which were conducted within two days after the QMCS post-test was given in class, students were asked to state their answer to each question and explain their reasoning out loud. Initially the interviewer intervened only to request that students talk more or to ask clarifying questions. After students had answered all questions, the interviewer asked more in-depth follow-up questions about issues raised in earlier responses and if requested, helped students with the questions they had answered incorrectly.

We conducted simulation interviews with six students: four from traditional courses and two who had taken the transformed course the semester before we created the simulation and were serving as LAs in the transformed course in the subsequent semester. In simulation interviews, there was an initial period of free exploration, in which students were asked to play with the simulation and talk out loud with little interference from the interviewer, followed by a period of guided exploration, in which the interviewer asked students to explore aspects of the simulation they had missed or asked follow-up questions about the concepts involved in the simulation.

All interviewed students were recruited by sending an email to the entire class and paying volunteers $\$ 20 / \mathrm{h}$ for their

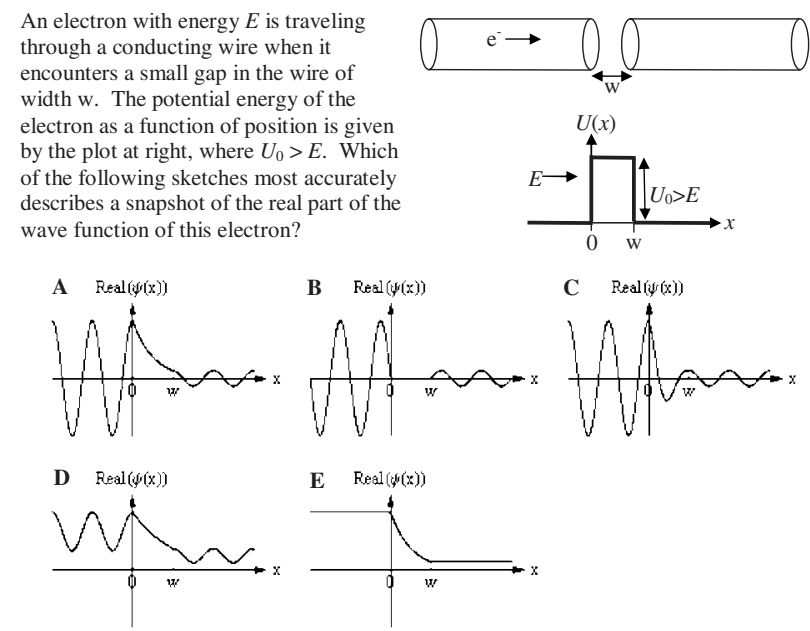

FIG. 6. A tunneling question from an early version of the QMCS. This question was developed to test students understanding of tunneling wave functions. It has been removed from the QMCS because interviews suggest that it tests memorization rather than conceptual understanding.

time. The payment ensured a greater diversity of volunteers than asking students to volunteer their time. (Some students stated that they were only there for the money and displayed little interest in the material.)

While our data include students working on many different problems in many different contexts, a large part of it is based on the questions shown in Figs. 6 and 7. In particular, the question in Fig. 7 was asked as a multiple choice question in some versions of the QMCS, which was given in class as an ungraded practice test before the final, as an essay question in which students were asked to "explain your reasoning" on the final exam one semester, as a multiple choice question on the final exam another semester, and in interviews for the QMCS and case study project.

By drawing on multiple forms of data, we have been able to track similar responses among many courses as well as looking at changes in student thinking as further transformations were introduced into the curriculum. When we noted interesting patterns in observations or interviews, we looked for corroborating evidence by reviewing videos and transcripts of interviews, field notes, and responses to online participation questions, homework, and exams.

\section{RESULTS}

Even in our transformed courses, we saw some evidence of most of the difficulties reported in previous research on student understanding of quantum tunneling. However, many of the previously reported difficulties were less prevalent or appeared in more subtle forms than we saw in traditional courses. We also saw many issues in our transformed courses that have not been previously reported.

\section{A. Difficulties drawing wave functions}

In a final exam question in the transformed course in Spring 2006, we asked students to draw the real part of the 
Suppose that in the experiment described in the previous question, you would like to decrease the speed of the electron coming out on the right side. Which of the following changes to the experimental set-up would decrease this speed?
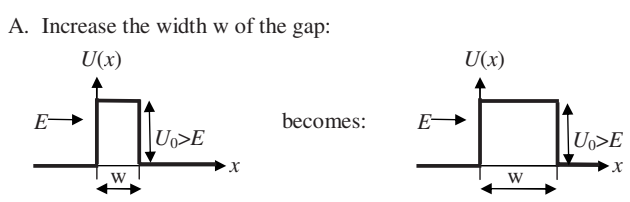

B. Increase $\mathrm{U}_{0}$, the potential energy of the gap:

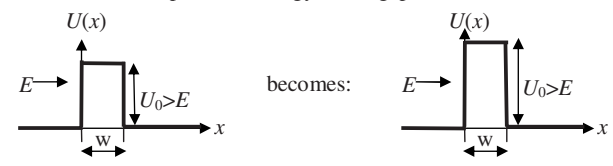

C. Increase the potential energy to the right of the gap:

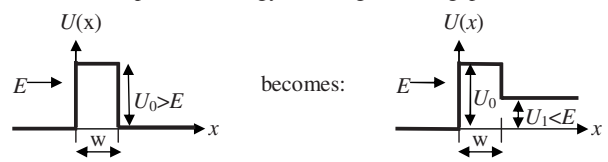

D. Decrease the potential energy to the right of the gap:

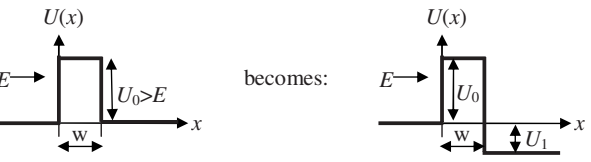

E. More than one of the changes above would decrease the speed of the electron.

FIG. 7. A tunneling question from an early version of the QMCS. This question was developed to explore the belief that energy is lost in tunneling. It has been removed from the QMCS because interviews suggest that responses from students in transformed courses are not necessarily indicative of whether students think energy is lost in tunneling.

wave function for an electron tunneling through an air gap in a wire (a square barrier of potential energy) and explain their drawings. We were looking for a drawing like that shown in Fig. 6(A) or a similar drawing of a tunneling wave packet. On this question, $18 \%$ of students drew the wave function with an offset as in Fig. 2(a) or 6(D) and 23\% drew a shorter wavelength on the right than on the left as in Fig. 2(b). While these difficulties, both of which have been reported on extensively in the previous literature, were fairly prevalent, we noticed in exam responses and in interviews with students in both traditional and transformed courses that the reasons for these two difficulties were not particularly deep. Students never volunteered any reasons for drawing the wave function in either of these ways, and when asked by interviewers to explain their drawings, they usually responded that they just remembered it looking like this. Figure 6 shows a QMCS question designed to test students' understanding of the wave function for a plane wave tunneling through a square barrier. We note that the percentage of students who answered this question correctly varied from semester to semester much more than other questions, and in interviews, students rarely gave any reasoning for either correct or incorrect answers but simply picked the picture that looked the most familiar or stated that they did not remember these pictures. In general, students' explanations of their answers to this question indicated that they were not reasoning through it at all but scanning for the picture that most closely matched their memories of figures from the lecture or textbook. Because this
TABLE I. Reasons students may think that energy is lost in tunneling. Reasons 1-3 are discussed in previous literature and reasons $4-6$ are new to the current study.

Reasons students may think energy is lost in tunneling

(1) Treating energy and wave function interchangeably

(2) Invoking dissipation

(3) Using the energy-amplitude relation for electromagnetic waves

(4) Confusion over how electron regains energy when it re-enters wire

(5) Treating total energy as a local characteristic of wave function

(6) Difficulty connecting energy to wave-function representation

question appears to test memory, rather than conceptual understanding, it has been removed from the QMCS.

\section{B. Energy loss: Another perspective}

In the transformed courses, because there was such a heavy emphasis on energy conservation, students quickly learned to say that energy is not lost in tunneling. When we asked them directly on exams whether "the total energy of an electron after it tunnels through a potential barrier is (a) greater than, (b) equal to, or (c) less than its energy before tunneling," between $70 \%$ and $93 \%$ answered correctly that it is equal. In homework, when students were asked "does an electron lose energy when it tunnels?" between $95 \%$ and $96 \%$ answered correctly that it does not and gave clear explanations of their reasoning, invoking the conservation of energy and the lack of dissipation. When the question in Fig. 7 was asked as an essay question on the final exam, only $7 \%$ of all students ( $31 \%$ of those who answered incorrectly) explicitly said that energy is lost in tunneling in their response, although a much larger percentage gave answers that implied energy loss.

In spite of students' correct answers on direct questions, energy loss in tunneling continued to be an issue. After the instruction described in Sec. IV, students asked repeatedly in lecture, problem-solving sessions, and online participation homework "why is the total energy the same after tunneling?" (These were often the same students who had given correct and clear answers to this question in earlier homework.) In questions about tunneling that did not directly ask about energy loss, some students continued to give answers that implied that energy is indeed lost in tunneling. Table I lists all the reasons we have seen students give for energy loss in interviews and in responses to an essay question on an exam. As discussed in Sec. III, reasons 1 and 2 are the standard reasons that have been given in most previous literature and reason 3 was postulated in a previous study. Reasons 4-6 are new to the current study.

In this section, we will outline the general results from the question shown in Fig. 7 and will then discuss the evidence for each of the reasons given for energy loss in Table I from interviews, observations, and student responses to an essay version of the question in Fig. 7 asked on a final exam. Unless otherwise noted, all interview quotes are from students in transformed courses. 
TABLE II. Percentage of students who selected each answer to the question shown in Fig. 7 in various courses. $N$ is the number of students.

\begin{tabular}{lcccccc}
\hline \hline Course & A & B & C & D & E & $N$ \\
\hline Traditional Engineering Spring 2005 (QMCS) & 18 & 10 & 24 & 15 & 33 & 68 \\
Traditional Physics Spring 2005 (QMCS) & 19 & 11 & 38 & 9 & 23 & 64 \\
Traditional Physics Fall 2005 (QMCS) & 12 & 11 & 38 & 15 & 24 & 54 \\
Traditional Physics Fall 2006 (QMCS) & 13 & 13 & 38 & 9 & 26 & 54 \\
Transformed Engineering Fall 2005 (QMCS) & 12 & 10 & 37 & 5 & 37 & 162 \\
Transformed Engineering Fall 2006 (QMCS) & 20 & 6 & 41 & 8 & 25 & 73 \\
Transformed Engineering Spring 2007 (QMCS) & 13 & 10 & 40 & 7 & 31 & 120 \\
Transformed Engineering Spring 2006 (exam & 2 & 3 & 58 & 5 & 31 & 177 \\
essay) & & & & & & \\
Transformed Engineering Spring 2007 (exam & 1 & 2 & 90 & 6 & 0 & 147 \\
multiple choice, after QMCS) & & & & & & \\
\hline \hline
\end{tabular}

The question in Fig. 7 was originally developed to test whether students think that energy is lost in tunneling. In order to answer this question correctly, students must recognize that total energy is constant and determine that since potential and kinetic add up to total energy, the way to decrease the kinetic energy on the right must be to increase the potential energy, as in the correct answer, C. The distracters $\mathrm{A}$ and $\mathrm{B}$ are very effective at eliciting the belief that energy is lost in tunneling, since students who think that energy is lost will usually think that more energy is lost in one or both of these cases. In interviews with students in traditional modern physics courses, we found that students' answers to this question were good indicators of whether they believed that energy is lost in tunneling; all students who did not choose the correct answer expressed the belief that energy is lost in tunneling. ${ }^{7}$

However, in later interviews with students in our transformed modern physics class, we found that even students who explicitly said that energy is not lost in tunneling sometimes chose incorrect answers, often for very subtle reasons. (Occasionally, students even argued for answers A and B by saying that no electrons will tunnel if you make the barrier sufficiently high or wide and if no electrons are coming out, you could say the speed is zero. While one could argue that these students were using questionable logic, their incorrect answers were not due to a misunderstanding of the physics.) We have also found that this question is much more difficult than other questions eliciting the idea of energy loss, with only $37 \%-58 \%$ of students answering correctly the first time they see it. Students' ability to answer this question also varies greatly depending on context. As shown in Table II, when the question was asked on the QMCS, an ungraded multiple choice conceptual survey that was used as a review for the final exam, $37 \%-41 \%$ of students in the transformed course for engineering majors answered correctly (higher than the scores in the traditional course for engineering majors and comparable to the scores in the traditional course for physics majors). However, when we gave it instead as an essay question on the final exam, asking students to explain your reasoning, 58\% answered correctly. We hypothesize that the process of explaining their reasoning led more stu- dents to figure out the correct answer; in interviews, we saw that many students initially answered with a variety of incorrect reasoning but in the process of attempting to explain their reasoning to the interviewer eventually came to the correct explanation. When we gave it as a multiple choice question on a final exam after asking it on the QMCS and reviewing it in class, so that students were already familiar with it, $90 \%$ answered correctly.

Because of the subtlety of the reasons students give for their answers and the context dependence of the scores, we no longer recommend the use of this question in multiple choice format as a diagnostic. However, we have found that it is extremely valuable for eliciting student thinking when used in an interview setting or as an essay question on exams.

\section{Reasons 1 and 2: Treating energy and wave function interchangeably and invoking dissipation}

In the transformed courses, the two standard reasons for energy loss did arise but were relatively infrequent. For example, when the question in Fig. 7 was asked as an essay question on the final exam, only $10 \%$ of all students (43\% of those who answered incorrectly) related the decrease in speed to the exponential decay of the wave function, implying that they were treating energy and wave function interchangeably (reason 1) and only $16 \%$ of all students $(67 \%$ of those who answered incorrectly) said that it requires more energy, or is harder, to tunnel through a wider or higher barrier, implying dissipation (reason 2).

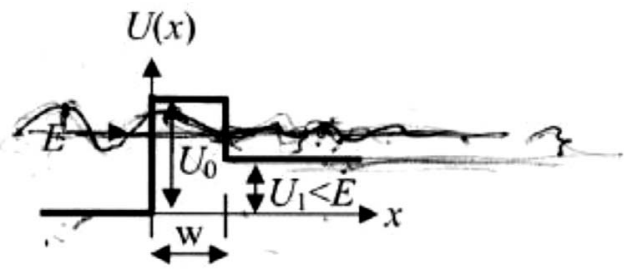

FIG. 8. A student drawing of a wave function on top of a potential-energy graph. 
Further, when students in interviews or problem-solving sessions seemed to have the common problems of treating energy and wave function interchangeably or thinking of dissipation, they usually corrected themselves without intervention from the interviewer or instructor. An example can be seen in an interview with a student who is struggling to answer the question in Fig. 7. The student begins with a typical response in which she interprets the height of the wave function as the kinetic energy:

It's either one of these two [A or B]. I'm just trying to think about it. I think it's this one right here [B] because it would-the wave function would come up here and then it would drop down a little bit but then it would keep going, and the distance between it and the potential energy would be the kinetic energy, kind of.... Well, no, this is-scratch that. We'll take it out. Because the energy has to be the same on both sides...

After she answered the question correctly, the interviewer asked her to explain what she was thinking before. She said:

Yeah. Um, I was thinking [pause] that a lot of times when I see these, I'm thinking of the wave function on top of it [draws wave function on top of energy graph-see Fig. 8], and I'm thinking of it dropping down a certain-dropping down, like, a certain rate depending on the difference between the energy-the electron's energy and the potential energy or the width. So I think about it that way. So I was thinking, once it - if it's coming up here and it drops down a little bit, it's gonna come up here on this side. And I'm kind of thinking, maybe, like, the amplitude of the wave function had to do with energy and so its distance from this potential was the kinetic energy or kind of could represent the kinetic energy. But then I wasn't too sure about that because I realized I was kind of thinking of the wave function instead of the energy so I had to, like, re-evaluate how I was thinking about it even though it kind of still works the same.

It is interesting that this student instinctively thought of drawing the wave function on top of the energy graph, although this semester, aside from in the question shown in Fig. 3, there were no pictures of a wave function on top of an energy graph in the lectures, textbook, or simulations. This example illustrates that eliminating such pictures, while helpful, is not sufficient to address the problem of students treating energy and wave function interchangeably.

Thus, in interviews, students in the transformed course were usually able to let go of the typical ideas that lead to belief in energy loss. However, these students often did say in interviews that energy is lost in tunneling. There are four further reasons they gave, all of which are distinct from the reasons most often given in the literature.

\section{Reason 3: Using energy-amplitude relation for electromagnetic waves}

In the transformed courses, the relationship between amplitude and energy for electromagnetic waves was very heavily emphasized in the section on the photoelectric effect in lecture, homework, and exams. Occasionally in interviews and observations of students in the transformed courses, but never in interviews with students in the traditional courses, students pointed out this relationship and asked why it was not the same for matter waves or assumed that it was the same. For example, in one interview, after a student had drawn a wave function with the same amplitude on both sides of the barrier "because the kinetic energy's the same, the total energy's the same, the potential energy's the same on each side," he corrected himself and explained "I was thinking more of the electromagnetic waves when I was thinking about that."

This reason was not very common and was only observed in relatively strong students. We mention it here mainly because it has been discussed in previous research. ${ }^{2}$

\section{Reason 4: Confusion over how electron regains energy when it re-enters wire}

One reason that some students give for energy being lost in tunneling appears to be associated with the particular physical example we use in class, that is, an electron traveling through a wire and tunneling through an air gap. Students can easily grasp the physical mechanism by which kinetic energy is lost when it goes from the first wire into the air gap. Earlier in the course, in the context of the photoelectric effect, we discuss the energy required to overcome the work function of the metal. While the photoelectric effect example is different from tunneling in that the kinetic energy of the electron does not become negative, it is similar in that kinetic energy is transferred to potential energy when an electron goes from one medium to another. Most students seem able to apply the concept of energy transfer from the photoelectric effect to tunneling, recognizing that the electron loses kinetic energy when it escapes the wire into the air gap. However, students do not understand the mechanism by which the electron regains its kinetic energy when it goes into the second wire. Therefore, while they know that energy is conserved, they express confusion over how the electron "gets back" the energy that went into overcoming the work function.

For example, one student, who was sufficiently bothered by this issue that she had asked her friends about it, said in an interview:

The kinetic energy starts at $E$ and then it drops down, takes energy to get up, and then it jumps back up to $E$. I talked to my friends. Why the hell they don't understand that exactly.

In another interview the following week, the same student brought up the issue again:

Yeah, because it takes energy to get out of metal, the work function. And it takes the amount of the potential energy - the barrier, this is the barrier's, so it uses that energy up and then it has a much slower-so it's going much slower. And then once it hits the other metal, hey, it's going fast again.... It's just weird, a little bit. You'd think it would slow down, but it is because the potential drops to zero again and conservation of energy, all the energy goes to kinetic. But it is a weird idea for me to think about. 
We have found that presenting students with a gravitational analogy of a ball rolling over a hill, in which the kinetic energy is lost as the ball rolls up the hill and regained as the ball rolls down the hill, helps students resolve this confusion. In every case we have seen, the main problem is that students are not able to apply the concept of converting potential energy to kinetic energy to this novel situation. However, they are quite comfortable with this concept in the context of gravitational potential energy, and once they are reminded of this familiar situation, they can apply the concept to the new situation. For example, after the interviewer suggested the analogy and asked why the ball regains its kinetic energy, the student quoted above said:

Because the gravitational force supplies it with energy. Going back down, like, the potential increases as you get farther and farther from the ground. When you're lifting something up, it gives it potential energy. And that's what - and as the ball rolls back down, it gains kinetic energy because it's going back down. So the force gives it energy, I guess. So maybe I'm just confused on what's the-hmm. [Pause] OK. Because I understand this really well. [Points to picture of ball on hill] But why the electron is or what the electron's doing and what-what's the force here that is giving it the energy. Here gravity and potential, I understand that. Maybe here I don't quite understand what's giving it back its-like, what's the force involved that's giving it back its energy, in a sense.... Yeah, I guess that's maybe my confusion. But when you look at it that way, it's really easy to understand. This is like a hill. It comes back down.

It is worth noting that this student traced her confusion back to not knowing what the force was. In quantum mechanics instruction, we typically ignore forces altogether and speak only in terms of potentials.

Some researchers are reluctant to use gravitational analogies in teaching quantum tunneling for fear that they may lead to the idea that tunneling involves a particle traveling through a physical barrier like a hill and exacerbate the difficulty of thinking that energy is lost due to dissipation. However, we have seen no evidence of such a link. Further, as Brookes and Etkina ${ }^{20}$ pointed out, the gravitational analogy is already inherent in the language we use, as seen in phrases such as potential step and potential barrier, and even in the word "tunneling." Even if we were careful to avoid such language, there is evidence that students have a tendency to interpret graphs too literally and think that higher on a graph means higher in space, regardless of context. ${ }^{39}$ The gravitational analogy is an important aspect of the expert model of tunneling. Therefore, we argue that it is preferable to address the strengths and limitations of the gravitational analogy directly rather than to avoid its use.

Another possible concern about the gravitational analogy is that the ball must have enough energy to get over the hill, and thus it is not actually tunneling. However, confusion over how the electron gets back its kinetic energy arose both in cases with the total energy less than the potential energy of the gap, as in tunneling, and in cases with the total energy greater than the potential energy of the gap, as in the first

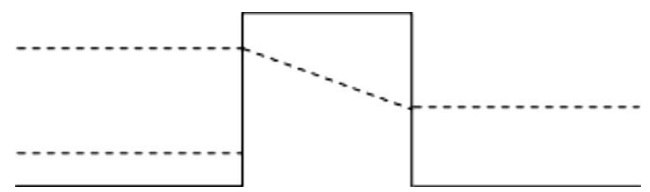

FIG. 9. A reproduction of a graph drawn by a student to represent the potential (solid line) and total (dotted lines) energies of a particle tunneling through a barrier. This student said the particle was losing energy because only a part of it was transmitted.

example in the tunneling tutorial. Thus, the difficulty does not seem to be particular to the case of the total energy being less than the potential energy of the gap, and we have not seen any problems as a result of the gravitational analogy not corresponding to tunneling.

Out of six students who were interviewed extensively on the relationship between the energy and wave function in tunneling, two exhibited this difficulty, and it was observed in students working on homework in problem-solving sessions. There is also some indirect evidence for this difficulty in some of the responses to the essay version of the exam question shown in Fig. 7: 11\% of all students (48\% of those who answered incorrectly) argued for option A and/or B by pointing out that the electron would be slower in the gap in these cases than in the original case. While it is possible that some of these students simply misread the question and thought it was asking how to slow the electron inside the gap, rather than to the right of the gap, it is clear from at least some of these responses that this is not the case. For example, "want to decrease its KE coming out. We can only do this by increasing the PE in order to borrow more KE from the system" and "a greater PE means a decrease in KE the $e^{-}$ will have once it merges on right side. Both B \& $\mathrm{C}$ would cause this result." These responses imply that students correctly understood that increasing potential energy reduces kinetic energy but did not recognize that the kinetic energy would increase again when the potential energy goes back down.

\section{Reason 5: Treating total energy as a local characteristic of wave function}

Another reason that students give for energy loss is that, since only part of the wave function is transmitted, only part of the energy is transmitted, with the rest being reflected. For example, when a student working on homework during a problem-solving session was asked to draw the potential and total energy of a tunneling electron, he drew a picture like the one shown in Fig. 9. He explained that the dotted line on the top left was the total energy of the incoming particle, which was then split into the reflected part (bottom left) and transmitted part (right).

Another student, who was attempting to answer the question in Fig. 7 in an interview, after explicitly stating that energy is not lost in tunneling, used a similar argument in an interview to justify her intuitive belief that the energy must be less on the right side of the barrier: 
Interviewer: ... does that mean that the total energy is going down when it goes through the barrier?

Student: The total energy is constant.

I: Ah! OK, so what energy is decreasing then, if it's not the total energy?

S: The energy - the energy-man-Well, OK. What I'm saying, but what I'm saying with caution-is- the energy of the wave function on this side-is decreasing. I want to make the energy of the wave function on this side decrease. But I'm also wary about that because- "the energy of the wave function on this side"? You know, the wave function is a wave function, and it has like parts to it, but it doesn't have like-No, it does-You can have a wave function like that... and it has a different energy here than it has here.

\section{I: Different total energy?}

S: No, total energy is of the entire wave function. What is total energy then? Is it this plus this plus this? Yikes! I need to study this for the final.

This difficulty appears to be caused by a lack of understanding of the fact that the total energy is a nonlocal property of the entire particle rather than a local function of position. This fact is not stressed in our class, nor in any class we know of, but perhaps it should be.

We note that in both examples discussed above, the problem involved a single electron tunneling, not an ensemble, and the students described it as such. Because our course did not discuss probability current, it is unlikely that this difficulty was due to students thinking of probability current or ensembles of electrons splitting. We further note that in both cases, students had been introduced to tunneling in terms of both wave packets and plane waves, so the difficulty cannot be attributed directly to either of these approaches.

While this difficulty has only been observed with the two students discussed above, these two examples are from courses in different semesters, taught by different instructors using different textbooks. Further, the problems they were working on were quite different and the two students had very different personalities.

\section{Reason 6: Difficulty connecting energy to wave-function representation}

Another reason that students give for energy loss in tunneling is related to the confusion between energy and wave function seen in reason 1 , but it is more subtle. In several interviews, we have observed students who explicitly state that the energy is not the same as the wave function but that the two must be related somehow, so the exponential decay of the amplitude must imply a loss of energy. These students are careful to distinguish between energy and wave function and do not use them interchangeably but believe that there must be some relationship between them. While reason 1 is simply a failure to distinguish between the two quantities, reason 6 is a search for a simple causal relationship that does not exist.
For example, when asked whether the probability of tunneling would change if the width of the barrier increased, a student said some things made him think that it would decrease and other things made him think that it would not change. When the interviewer asked what made him think it would not change, he said:

Well, it would be diagrams like this. [Points to energy graph] One thing that the text doesn't really havedoesn't focus nearly as much as you guys do in the course, and I don't know if that's good or bad, are these diagrams. You guys use these diagrams a lot, which is great. The text doesn't so much, it sort of approaches it in a little different way. So if we are to evaluate these diagrams, put our total energy line in, evaluate how that corresponds with our potential energy, you - it sort of-[pause] maybe this forces me to think too much about energies. For example-I mean, that's more classical physics, is it not? If the particle has sufficient energy to get to the other side. Quantum's a whole other story where we're not talking about so much energies. We are, but we're also talking about probabilities, correct? So there's sort of two ways to think about this and maybe that's why I'm a little confused still, at this late date.

Another student, when asked whether the probability of tunneling would change if the initial energy of the incoming particle decreased, said:

The amplitude shouldn't be affected by the energy other than its exposition. Yeah. I think. And then-I believe it's still gonna do the exponential decay. [Draws] OK. So now-OK, so, hmm, probability of the electron tunneling through the barrier. The difference between the total energy and the potential energy of the gap is larger now, so I would say-I feel like, um, that would mean that it has less of an opportunity, less chance, less probability of it tunneling through. What am I trying to say here? When an electron has to convert a certain amount of kinetic energy to come out of a wire to potential energy, and in this case it has to convert this much [points] or this much will be potential energy, that difference there, which is more than the original case. So [pause] I don't know. Um, I'm not quite figuring out how to connect it. But the larger difference between the total energy and the potential energy of the gap I think has something to do with the probability of the electron tunneling through or not, compared to the first.

This difficulty reveals why emphasizing that the wave function and energy are not the same thing is not sufficient to address the student belief that energy is lost in tunneling. Even if students realize that energy and wave function are not literally the same thing, they struggle to make connections between these two quantities that are emphasized in the study of quantum mechanics. One quantity, the wave function, is wholly unfamiliar to students, and the other quantity, energy, is treated in an unfamiliar way: graphed as a function of position but applied to a delocalized object.

Out of six students who were interviewed extensively on the relationship between the energy and wave function in 
tunneling, four exhibited some form of this difficulty. It was also observed in students working on homework in problemsolving sessions. It also came up in class when students were working through the tunneling tutorial. Although the tutorial specifically said not to worry about the relative magnitudes when sketching wave functions, many students asked how to figure out the amplitude from the energy and expressed frustration that they could not find a connection.

Unlike the previous three difficulties, this was also observed in students in the traditional courses. For example, one student from a traditional course, when asked how the wave function is related to the energy, replied, "I can't remember. I wish I did. But I can swear, well not swear, but I can almost remember my professor saying that the energy is encoded in the wave function, somehow, I can't remember exactly now."

\section{Giving potential energy a physical context}

One conclusion of our study is that understanding the context of potential-energy graphs is a difficult task for students, and a great deal of instruction is needed to address this issue. In a previous study ${ }^{7}$ we reported on interviews with students in the traditional modern physics for engineering majors course in Spring 2005. Students in this course had no idea what the potential-energy graphs mean. In the transformed courses, we observed that students still struggled with the basic meaning of potential-energy graphs, but as we refined our curriculum, their questions about these graphs became more sophisticated, illustrating a struggle to relate the graphs to physical reality in a deep and meaningful way. The extent of questioning from students in the transformed course indicates what a difficult subject this is and how hopeless it is to expect students to build meaningful models of these graphs if the course does not explicitly help them do so.

In Fall 2005, the first semester of the transformed course, even after focusing on the physical context of potential and explicitly addressing possible confusion arising from sloppy language in the text using potential and potential energy interchangeably, students expressed a great deal of confusion over the meaning of potential energy. In weekly online extra credit, we asked students to submit any unanswered questions they had about the course material. Here is a sample of these questions regarding potential energy:

(i) "I get very confused by exactly what an infinite well is. What is it, how is it infinite? do we just make it that way?"

(ii) "I have trouble understanding what the potential is when we are looking at models of an electron in a wire, free space, finite square well, infinite square well. I am sort of getting this idea of it being similar to a work function in that once the potential $(V)$ is less than the potential energy, the electron is out of the wire. I can usually follow the math/calc that follows the examples okay, but the overall concept of this potential $(V)$ still confuses me, and so I still don't have a firm grasp of [what] the square well models mean/represent/ whatever."

(iii) "I cant find a general description of an infinite well, i understand what it does but not what it is or where its used."

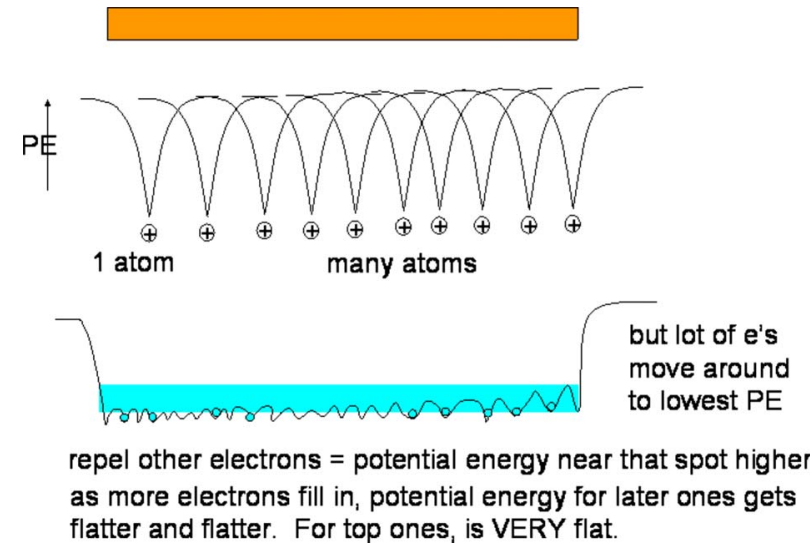

FIG. 10. (Color) An illustration of how to build up a square well by adding up the Coulomb wells of individual atoms (taken from slides used in lecture in Spring 2006, Fall 2006, and Spring 2007). This is a simplified sketch in which the discontinuities of the potential at the ion cores are smoothed over and irrelevant details such as the zero of potential are not labeled.

(iv) "Voltage is used when we talk about electromagnetic forces, like the coulomb force. What I'm confused about is that we used a voltage well to show the strong force in effect. Is it accurate to show the strong force as a very deep voltage well?"

The first three students were struggling to make sense of what the potential-energy diagram for an infinite square well means and how it relates to a real physical system. The last student thought that the symbol $V$ that we used for potential energy represented voltage, although we had pointed out repeatedly in class that it did not.

Further evidence for student confusion about potential energy can be seen in our observations of students' responses to the first question of our tunneling tutorial, which asked students to draw the potential energy as a function of position for an electron traveling through a long copper wire and tunneling through an air gap [see Fig. 4(b)]. At this point in the course, students had worked extensively with a square well as a representation of the potential energy of an electron in a wire but had not previously seen this example of a wire with an air gap. We expected them to use their knowledge of the potential energy of individual wires to draw a square barrier for this new situation. While many students did draw the correct potential energy, we observed that many students got it backward, drawing a well instead of a barrier. A common student explanation for the well was that the air gap was a "hole" and therefore should be represented by a well. This response betrayed a lack of understanding of why a well represents the potential energy of the wire. In subsequent semesters, we added instruction before the tutorial on how to build up a square well by superimposing the Coulomb potentials of all the individual atoms that make up a wire. As illustrated in Fig. 10, adding the potentials of all the ion cores that make up a wire produces a potential similar to a square well but with a dip at each atom. Since the electrons are mobile and attracted to these dips, adding in the potentials of the electrons tends to smooth out the potential to make it even more like a square well. After this instruction, 
anecdotal observations indicated that fewer students drew a well instead of a barrier in the tutorial. In later semesters, we also demonstrated this concept with the Quantum Bound States PhET simulation.

Students still struggled to relate the potential-energy graph to reality to the extent that some viewed the graph and the electron in the wire as describing two different things. Here is an example from an interview in which a student was trying to figure out how the width of the barrier affects the probability of tunneling:

But I don't know if it explained as well as it needed to or if I just didn't understand as well as I needed to whether width [holds out thumb and forefinger to indicate width of space between them] meant actual real classical physics width [holds hands out to indicate width of space between them] or more theoretical width [points to potential barrier on sheet], which is like the-which might be more represented here.

Another example of a student struggling with potentialenergy graphs can be seen in a student who asked a question after class that revealed that he was misinterpreting the pictures in Fig. 4 to mean that the wire was sitting on top of the potential energy.

Students also worried about the applicability, limitations, and relevance of the model of the square well for an electron in a wire. For example, students frequently asked about collisions with the atoms in the wire and whether these would constitute measurements of the electron and localize it. In discussing tunneling, they struggled with the concept of infinitely long wires and frequently discussed the reflection of the electron when it reached the end of the wire. While working through the tunneling tutorial, one student asked why the electron would flow from one wire to the other if there was no potential difference between the two wires. The answer to this question is that you would not have a net flow of electrons from left to right without a potential difference but that electrons would constantly flow back and forth due to thermal energy. This student also asked whether you could really measure a single electron flowing through a wire and why we were studying it if you could not. He was satisfied only after a long explanation of how you could predict net current by adding up the effects of single electrons. This example demonstrates that even with a physical context, a square barrier with an equal potential energy on either side (the prototypical system used in the standard presentation of tunneling) is still artificial because in reality a net current does not flow without a voltage between the two sides of the barrier. These questions further demonstrate that physical context is important not just for giving the material relevance but for conceptual understanding of the material itself.

Student difficulties with potential energy can also be seen in the questions they asked during the section on the applications of tunneling, which included alpha decay, scanning tunneling microscopes, and getting shocked when you rub your foot on the carpet and approach a metal doorknob. We asked students to figure out the potential-energy graphs for each of these applications, shown in Fig. 11, using a series of concept questions in lecture as well as more detailed questions in homework. Determining each of these potential-

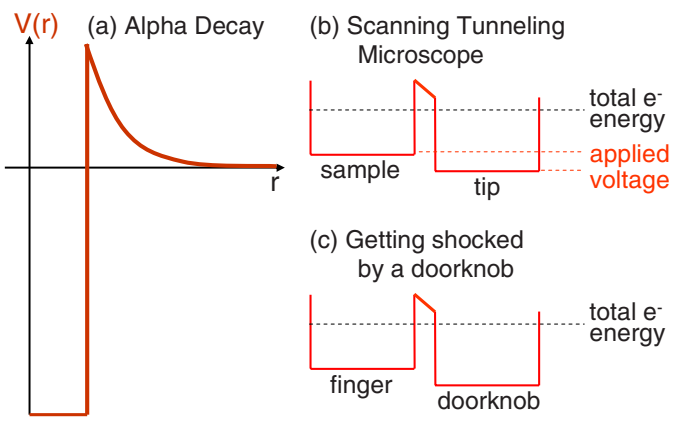

FIG. 11. (Color) Potential-energy graphs for (a) an alpha particle undergoing alpha decay, (b) an electron in a scanning tunneling microscope, and (c) an electron in your finger when you get shocked by a doorknob. Determining how to draw each of these graphs requires many subtle approximations.

energy graphs requires many subtle approximations, which may not be apparent until one is faced with a barrage of student questions. For example, to determine the potentialenergy graph for alpha decay, one must approximate the strong force as a flat potential throughout the nucleus, although there is no model in nuclear physics that predicts such a potential, one must recognize that the strong force dominates in the nucleus and the Coulomb force dominates outside, and one must treat the alpha particle that is going to be ejected as having an independent existence and a welldefined energy prior to decay. Gurney and Condon ${ }^{40}$ explicitly discussed all of these approximations in their 1929 paper explaining radioactivity on the basis of tunneling. Yet most textbooks simply give such graphs without explanation.

The following questions from students illustrate that our students struggle with these approximations:

(i) "How do the Coulomb force and the strong force relate to each other?"

(ii) "How do you find the distance where the strong force takes over?"

(iii) "Is the potential really square like that?"

(iv) "Do alpha particles already exist in the nucleus or are they created upon radioactive decay?"

In the first two questions, students are struggling with the assumption that the strong nuclear force dominates in the nucleus and the Coulomb force dominates outside of it. The last two questions illustrate the simplifications required to come up with a solvable model.

Similar questions illustrated students' struggles to understand the potential-energy graph for a scanning tunneling microscope:

(i) "As the electrons tunnel through, isn't the sample potential energy going to drop?"

(ii) "The quantum tunneling microscope can be used on any material even though not every material has a "sea" of electrons? Wouldn't losing an electron in a crucial covalent bond break the molecule apart?"

The answer to the first question is that the potential energy would drop if the sample were not hooked to a voltage supply to keep the voltage constant. This student missed the function of the voltage supply, but the question illustrates that he was thinking carefully about the physical system. He also recognized that the behavior of the electrons could ac- 
tually change the overall potential energy, a fact which is never discussed in the standard presentation, where the potential-energy function is taken as a given. The answer to the second question is that scanning tunneling microscopes do not work on insulators, an issue that is never discussed in modern physics courses, but is the focus of a recent Nature article. $^{41}$

In spite of all these difficulties throughout the course, when we asked students to explain the physical meaning of the potential-energy graph of a square barrier on a homework question toward the end of the last two semesters, nearly all gave clear and correct explanations and related the graphs to a real physical context. Further, from interviews with 24 students in the transformed courses, there was only one case in which a student treated the potential-energy graph as an external thing unrelated to the potential energy of the electron, as we saw consistently in interviews with students in a traditional course in an earlier study. ${ }^{7}$ This case was so exceptional, especially because it was a particularly good student (he received an $\mathrm{A}$ in the course), that the interviewer asked him afterwards if he had done the tunneling tutorial. He said he had been busy that week and skipped it and jokingly commented, "In conclusion, that's a good assignment because you should listen to this guy try to explain it."

\section{Plane waves}

Plane waves cause further barriers to student understanding. While plane waves are mathematically simple, conceptually it is quite difficult to imagine a wave that extends forever in space and time, especially when it is tunneling. The language we use to describe tunneling is time dependent. For example, we say that a particle approaches a barrier from the left and then part of it is transmitted and part of it is reflected. This language is difficult to reconcile with a picture of a particle that is simultaneously incident, transmitted, and reflected for all time. The following student quote, from a homework question asking what questions students still had about tunneling after instruction, illustrates the kind of confusion created by using plane-wave solutions:

Say you have two finite lengths of wire very close together. I don't really see how we assume the electron is in one wire, get a solution, then use that to determine psi across the gap and then use that to determine the probability that the electron is in the other wire. Over time don't the probabilities even out (i.e., we have no clue which wire the electron's in)?

This student is actually struggling with two common issues for students: confusion over the physical meaning of plane waves and concern over what happens when the electron gets to the end of the wire. Many students have trouble with the idea of wires extending to infinity and talk about the electron waves reflecting off the end of the wire, interfering with themselves and creating a big mess. This is physically accurate, but outside of the realm of standard treatment, which assumes that wires do not have ends.

In student interviews to test the usability and effectiveness of the Quantum Tunneling and Wave Packets simulation, we saw that students were much more comfortable with the wave-packet representation than the plane-wave representation. We conducted interviews with six students, all of whom had completed either a transformed (2) or traditional (4) course in modern physics. These students were asked to explore the simulation and think out loud. The interviews started with free exploration, followed by questions from the interviewer about aspects of the simulation that the students had not explored on their own. All students discovered plane-wave mode on their own (the simulation starts in wave-packet mode), but four out of six switched back to wave-packet mode immediately and the other two only explored it for a few minutes before switching back. Two students switched back without comment, one commented that the plane wave was "too unrealistic," one commented that he did not remember what a plane wave was and was more familiar with a wave packet, and one commented, "that's definitely a visualization I didn't think of." Only one student commented that plane-wave mode made sense. Some students did eventually return to plane-wave mode in order to explore specific features, but all students spent most of the free exploration time in wave-packet mode and quickly returned to wave-packet mode after answering the interviewer's questions about plane-wave mode. One student, after trying it and switching back without comment, who did not use plane-wave mode again until the interviewer asked him to explore it, said he had forgotten about it.

It is possible that these students' clear preference for wave packets over plane waves was merely due to the wave-packet representation being more visually appealing. However, we note that students were able to use the wave-packet mode and learn new physics from it, in spite of its complexity.

Because the Quantum Tunneling and Wave Packets simulation provides such a compelling visual representation, it immediately brings to the surface several troubling issues regarding plane waves that are swept under the rug in standard treatments of tunneling because textbooks focus on only a few special cases in which these issues are not apparent.

According to the standard probability interpretation of the wave function, the reflection and transmission probabilities should be given by the area under the reflected and transmitted parts of $|\psi|^{2}$, respectively, divided by the area under the incident part of $|\psi|^{2}$. Since all these areas are infinite, one cannot calculate the reflection and transmission probabilities as one would naively expect. It is quite tempting (and quite wrong) to assume that the infinite widths simply cancel and that relative amplitudes should be a good indication of relative probabilities. However, this is not necessarily the case for plane waves, and the simulation reveals that there are even cases in which the amplitude of the transmitted wave is larger than the amplitude of the incident wave (see Fig. 12). This is such a surprising result that many experts, when they first see such a case, think there is a bug in the simulation.

Students often cue off the amplitude of the plane wave as a measure of probability and draw incorrect conclusions. In observations of students attempting to calculate reflection and transmission coefficients during problem-solving sessions, we noticed that many students initially assumed that they were given by 


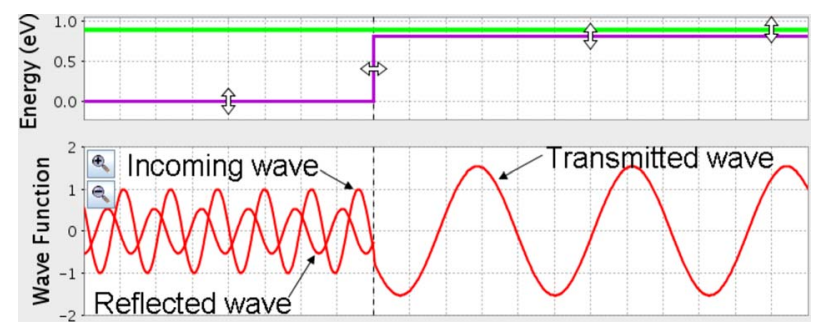

FIG. 12. (Color) A case where the amplitude of the transmitted wave is higher than the amplitude of the incident wave.

$$
R=|B|^{2} /|A|^{2}
$$

and

$$
T=|C|^{2} /|A|^{2},
$$

where $A, B$, and $C$ are the amplitudes of the incident, reflected, and transmitted waves, respectively. These equations happen to be correct for plane waves tunneling through a square barrier with the same potential on both sides, since the particle speeds happen to cancel, but Eq. (2) is wrong for a step potential or for any other situation in which the potential is different for the incident and transmitted waves.

Thus, both faculty and students tend to assume that the amplitude alone is an accurate indicator of probability and make mistakes as a result. Yet most textbooks quickly gloss over this issue. Most quantum mechanics textbooks simply state that the reflection and transmission coefficients for plane waves are determined by the probability current, without explaining why it is necessary to introduce this concept here and not elsewhere. (One textbook ${ }^{24}$ justifies defining $R$ and $T$ in terms of probability fluxes by saying that it is done "by accepted convention" in order to ensure that $R+T=1$.) In many modern physics textbooks, this issue is not discussed at all, and the equation for the transmission coefficient is simply given either in terms of particle velocities $(v)$ or wave numbers $(k)$,

$$
T=\frac{v_{t}|C|^{2}}{v_{i}|A|^{2}}=\frac{k_{t}|C|^{2}}{k_{i}|A|^{2}}
$$

(the subscripts $i$ and $t$ denote the incident and transmitted waves, respectively), with no explanation of where the factors of $k$ or $v$ come from. Some textbooks simply give Eq. (2) for the transmission coefficient, with no mention that this applies only for the special case in which $v_{t}=v_{i}{ }^{42-47}$ While we understand that the authors of these textbooks are attempting to avoid excessive mathematics that would obscure the basic concept of tunneling, presenting the transmission coefficient only for this special case leads students to draw many incorrect conclusions when attempting to extend their knowledge to other contexts.

We know of only two textbooks that give further justification by deriving the probability for a wave packet in the limit that the width goes to infinity. ${ }^{48,49}$ However, even in these books, it is not intuitively clear why an infinitely wide wave packet should lead to a probability proportional to the particle speed. We recommend an alternative treatment suggested by Lande et al.,${ }^{50}$ in which reflection and transmission coefficients are derived from wave packets, demonstrating that the factor of the $v$ results from the fact that the widths of the reflected and transmitted wave packets are a function of the speed at which they move in their respective media. This derivation is more intuitive than the derivation from probability current both because it relates more easily to the typical definition of probability as it relates to the amplitude of the wave function and because wave packets are more physical than plane waves.

A second problematic issue that is often swept under the rug is the issue of wave speed vs particle speed. Because the treatment of waves is being pushed out of the physics curriculum at many institutions, many students do not know the difference between phase velocity $\left(v_{\phi}=\omega / k\right)$ and group velocity $\left(v_{g}=d \omega / d k\right)$. For a Schrödinger wave function, the phase and group velocity are given by

$$
\begin{gathered}
v_{\phi}=\frac{\hbar k}{2 m}+\frac{V}{\hbar k}, \\
v_{g}=\frac{\hbar k}{m} .
\end{gathered}
$$

While the velocity of a particle corresponds to the group velocity of its wave function, the only velocity apparent in the visual representation of a plane wave is the phase velocity. The distinction causes confusion when the potential energy changes. Students can see in the simulation that if they increase the potential energy, the "wave speed" increases, which seems to contradict their intuition that increasing the potential energy should decrease the kinetic energy, and therefore the speed (since $K E=E-V$ ). In fact, increasing the potential energy increases the phase velocity or wave speed but decreases the group velocity or particle speed. The only way we know to gain any physical intuition for the group velocity of a plane wave is again to imagine it as an infinitely wide wave packet, in which case the group velocity is the speed at which that wave packet travels.

The distinction between wave speed and particle speed also causes problems in trying to explain why the probability is proportional to the current and not simply to the square of the amplitude of the wave function. As discussed above (see Fig. 12), the transmitted amplitude can be larger than the incident amplitude if the transmitted particle speed is smaller. However, in all such cases, the wave speed is actually larger, so it appears that the transmitted wave has larger amplitude and is moving faster, obscuring the correct explanation, that it has a smaller particle speed to compensate for the larger amplitude.

We point out these issues so that instructors will be aware of the complexities inherent in discussing plane waves and consider the advantages of focusing on more realistic wave packets. We do not have solutions for how to address the difficulties with plane waves (aside from avoiding plane waves and focusing on wave packets), and we hope that other researchers will pursue these questions further. 
(a)

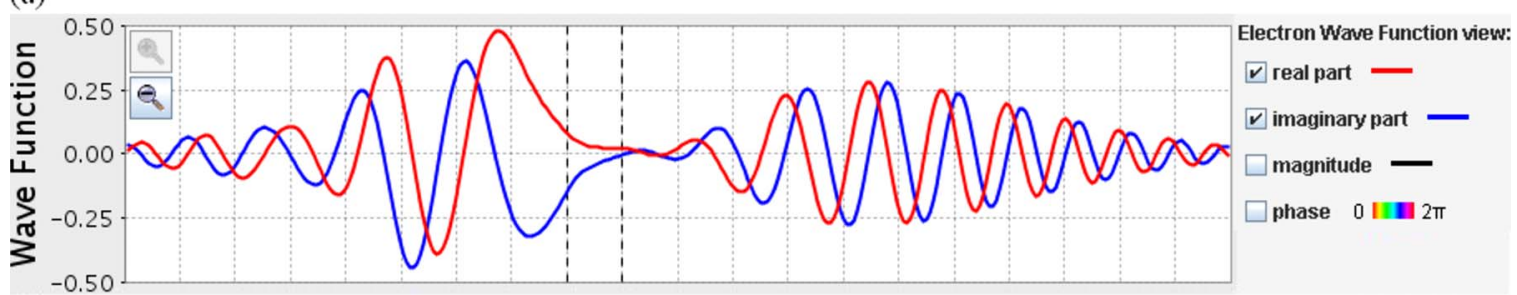

(b)

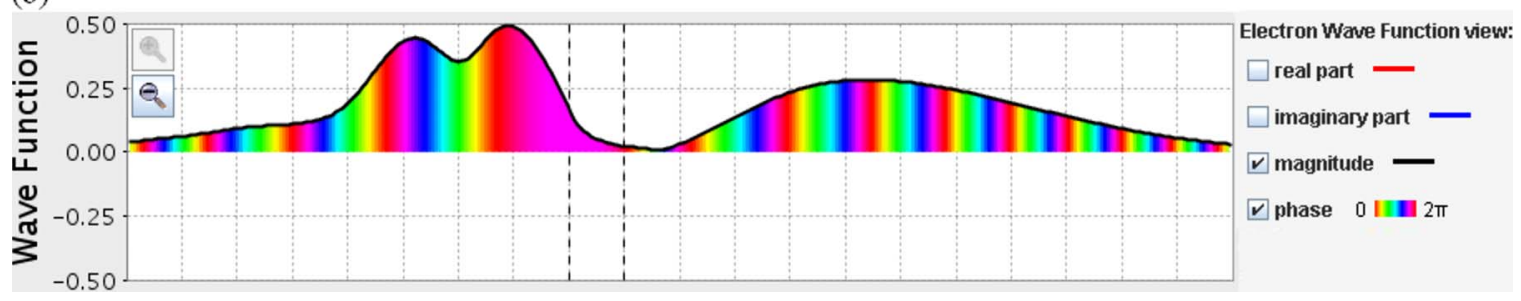

FIG. 13. (Color) (a) A representation showing the real and imaginary parts of a wave function and (b) a representation showing the magnitude and phase of a wave function. In interviews we see that students can make sense of representation (a) but not representation (b).

\section{E. Representations of complex wave functions}

Students often have difficulty understanding the meaning of complex wave functions. This can perhaps best be illustrated by the observation that students frequently ask, "What is the physical meaning of the imaginary part of the wave function?" but never ask about the physical meaning of the real part, even though both have the same physical significance.

Ambrose $^{1}$ found that some students believe that the wave function is only "real" in classically allowed regions, so that the real part is zero inside the barrier. We saw this problem in one interview.

All of the textbooks used in courses in this study regularly plotted only the real part of the wave function but referred to it as "the wave function," as in Fig. 1. In the QMCS and in the transformed courses, we always labeled such pictures explicitly as the real part. However, we found in interviews that even students in the transformed courses who had seen explicit discussion of both the real and imaginary parts were often confused by requests to draw "the real part of the wave function." When asked to draw the real part of the wave function on the exam question discussed at the beginning of Sec. VI, six students (3\%) said that the wave function is only real inside the barrier and set it to zero everywhere else.

To address these problems, we designed the Quantum Tunneling and Wave Packets simulation (as well as two other PhET simulations on quantum wave functions, Quantum Bound States and Quantum Wave Interference) to include both the real and imaginary parts on an equal footing [see Fig. 13(a)] and to include time dependence so that students could see how the wave function alternates in time between the real and the imaginary parts. For completeness, we also included the "phase color" representation used exclusively in most non-PhET simulations of wave functions, in which a curve representing the magnitude of the wave function is filled in with colors representing the phase [Fig. 13(b)].

In interviews with five students on Quantum Wave Interference, ${ }^{51}$ one student commented that he did not understand real and imaginary numbers and one student wondered why the imaginary part did not look different from the real part until he paused the simulation and could see that they were out of phase. Aside from these two, whose confusion stemmed more from their expectations than from the simulation, the students interviewed did not express any confusion over the real and imaginary representations of the wave function in interviews on Quantum Tunneling and Wave Packets and Quantum Wave Interference. Several students also learned important concepts by playing with the real and imaginary views. For example, students figured out from the simulation that the real and imaginary parts were $90^{\circ}$ out of phase and that the real and imaginary parts add up to a constant probability density in an energy eigenstate even though each individual component changes in time.

On the other hand, the phase color representation caused significant problems for most students. In interviews on Quantum Wave Interference, three out of five students interviewed explored this view. None of the three made any comments on it on their own aside from one student who said it hurts his eyes, so the interviewer asked them what it was showing. One student said it was "some sort of frequency type of thing" and speculated that teal would constructively interfere with teal and destructively interfere with the opposite of teal. Another stared at the screen in confusion for over a minute and then described it as "some sort of representation of both the real part and the imaginary part" showing that "pink is areas of high real part and low imaginary part or something?" Another student was unable to give any explanation. When the same three students were interviewed later on Quantum Tunneling and Wave Packets, the two who had given explanations in earlier interviews did not comment on phase view again. The student who had been unable to give any explanation remembered that this view had been used in his quantum course but still could not explain what it meant. Of three additional students who were interviewed on Quantum Tunneling and Wave Packets but not Quantum Wave Interference, two expressed frustration over the phase view and were unable to explain it, and the third, when asked to explain it, said only that it showed "something about wavelength." When given a choice, none of the students spent 
much time in phase mode, returning quickly to real or magnitude mode after answering the interviewer's questions.

Phase color is still an option in the simulations for instructors who would like to explicitly teach the use of this representation or use activities developed for other simulations. However, based on our interviews, we do not recommend the use of the phase color representation with students.

\section{F. "Hard questions"}

One striking result of our transformed instruction was the number of student questions probing the relationship of the course material to reality, many of which were sufficiently difficult that most expert physicists could not easily answer them. Many examples of these questions have already been discussed in Sec. VI C. Below are some further examples:

(i) What [happens if the electron is spread out] in the wire and you cut the wire in half?

(ii) How come we don't count the position in the wire? How come we only count the energy?

(iii) Wouldn't there be a charge difference in the wire if it were more likely to be found in the center?

(iv) If everything's got to be measured for it to be localized, how come everything's already localized? I'm not going around measuring things.

We hypothesize that these questions are a result of the combination of interactive engagement techniques with a focus on real-world applications. Our students are constantly engaged in a struggle to relate the material to reality. We regard the quantity of such questions as a sign that this struggle is very difficult. We question whether there is much learning in courses where students are not asking such questions.

\section{LESSONS FOR IMPROVING STUDENT LEARNING OF QUANTUM TUNNELING}

Our research demonstrates that a focus on addressing common student difficulties is helpful, but not sufficient, for improving student learning of quantum tunneling. By addressing these difficulties and focusing on relating the mate- rial to reality, we have uncovered deeper problems in students' ability to use the basic models of quantum mechanics, such as wave functions as descriptions of physical objects, potential-energy graphs as descriptions of the interactions of those objects with their environments, and total energy as a delocalized property of an entire wave function that is a function of position. We have found that real-world examples are useful not just to help students see the connection to their lives but also to help them make sense of the models they are using.

Effective curriculum on quantum tunneling must explicitly help students learn to build these models. Two practices that we have found useful are focusing on how to relate potential-energy graphs to physical systems and starting with wave packets rather than plane waves.

There are several further practices that, although we have not tested them on a large scale, our research suggests would be valuable. These include (1) tutorials to lead students through the process of drawing potential-energy graphs for various physical situations, ${ }^{52}$ (2) explicit discussion of the strengths and weaknesses of gravitational analogies, (3) explicit discussion of the reasons for the focus in quantum mechanics on an energy representation rather than the force representation used in introductory physics, and (4) explicit discussion of why total energy is quantized (for bound particles) but potential energy is not.

\section{ACKNOWLEDGMENTS}

We thank Chris Malley, the software engineer for the Quantum Tunneling and Wave Packets simulation, as well as Mike Dubson and Sam Reid for help with the physics and numerical methods involved in the simulation. We thank Michael Wittmann for convincing us to write this paper and Travis Norsen, Mike Dubson, and Chandra Turpen for useful discussions and feedback. We also thank the PhET team and the Physics Education Research Group at the University of Colorado. This work was supported by the NSF, The Kavli Institute, The Hewlett Foundation, and the University of Colorado.
${ }^{1}$ B. Ambrose, Ph.D. thesis, University of Washington, 1999.

${ }^{2}$ L. Bao, Ph.D. thesis, University of Maryland, 1999.

${ }^{3}$ J. T. Morgan, M. C. Wittmann, and J. R. Thompson, in 2003 Physics Education Research Conference Proceedings, edited by S. Franklin, K. Cummings, and J. Marx (AIP, Melville, NY, 2004).

${ }^{4}$ M. C. Wittmann, J. T. Morgan, and L. Bao, Addressing student models of energy loss in quantum tunneling, Eur. J. Phys. 26, 939 (2005)

${ }^{5}$ J. Falk, M.S. thesis, Uppsala University, 2004.

${ }^{6}$ D. Domert, C. Linder, and Å. Ingerman, Probability as a conceptual hurdle to understanding one-dimensional quantum scattering and tunneling, Eur. J. Phys. 26, 47 (2005).

${ }^{7}$ S. B. McKagan and C. E. Wieman, in 2005 Physics Education
Research Conference Proceedings, Exploring Student Understanding of Energy through the Quantum Mechanics Conceptual Survey, edited by P. Heron, L. McCullough, and J. Marx (AIP, New York, 2006).

${ }^{8}$ S. B. McKagan, K. K. Perkins, and C. E. Wieman, in 2006 Physics Education Research Conference Proceedings, Reforming a large lecture modern physics course for engineering majors using a PER-based design, edited by L. McCullough, P. Heron, and L. Hsu (AIP, New York, 2007).

${ }^{9}$ Course materials available online at http://per.colorado.edu/ modern

${ }^{10} \mathrm{D}$. Hestenes, Toward a modeling theory of physics instruction, Am. J. Phys. 55, 440 (1987).

${ }^{11}$ A. P. French and E. F. Taylor, An Introduction to Quantum Phys- 
ics (Norton, New York, 1978).

${ }^{12}$ A. Goldberg, H. M. Schey, and J. L. Schwartz, Computergenerated motion pictures of one-dimensional quantummechanical transmission and reflection phenomena, Am. J. Phys. 35, 177 (1967).

${ }^{13}$ It is linguistically awkward to speak of the potential energy "inside the barrier" since the potential energy is the barrier, but it is important to be explicit, as many students do not recognize the equivalence of potential energy and barrier.

${ }^{14}$ L. Viennot, Spontaneous reasoning in elementary dynamics, Eur. J. Sci. Educ. 1, 205 (1979).

${ }^{15}$ D. E. Trowbridge and L. C. McDermott, Investigation of student understanding of the concept of velocity in one dimension, Am. J. Phys. 48, 1020 (1980).

${ }^{16}$ D. E. Trowbridge and L. C. McDermott, Investigation of student understanding of the concept of acceleration in one dimension, Am. J. Phys. 49, 242 (1981).

${ }^{17}$ J. Clement, Students' preconceptions in introductory mechanics, Am. J. Phys. 50, 66 (1982).

${ }^{18}$ I. A. Halloun and D. Hestenes, Common sense concepts about motion, Am. J. Phys. 53, 1056 (1985).

${ }^{19}$ I. Galili and V. Bar, Motion implies force: Where to expect vestiges of the misconception? Int. J. Sci. Educ. 14, 63 (1992).

${ }^{20}$ D. T. Brookes and E. Etkina, in 2005 Physics Education Research Conference Proceedings, edited by P. Heron, L. McCullough, and J. Marx (AIP, Melville, NY, 2006).

${ }^{21}$ D. T. Brookes, Ph.D. thesis, Rutgers University, 2006.

${ }^{22}$ M. C. Wittmann, R. N. Steinberg, E. F. Redish, and University of Maryland Physics Education Research Group, Activity-Based Tutorials Modern Physics (Wiley, New York, 2005), Vol. 2.

${ }^{23}$ R. Harris, Nonclassical Physics, 1st ed. (Pearson, Menlo Park, 1998).

${ }^{24}$ R. Eisberg and R. Resnick, Quantum Physics of Atoms, Molecules, Solids, Nuclei, and Particles (Wiley, New York, 1985).

${ }^{25}$ The simulation discussed in the next section uses both labels to help students relate the more correct term to the term that is more commonly used in textbooks.

${ }^{26} \mathrm{http}: / /$ phet.colorado.edu/tunneling

${ }^{27}$ http://phet.colorado.edu

${ }^{28}$ J. R. Hiller, I. D. Johnson, and D. F. Styer, Quantum Mechanics Simulations: The Consortium for Upper-Level Physics Software (Wiley, New York, 1995).

${ }^{29}$ B. Thaller, Visual Quantum Mechanics (Springer, New York, 2000).

${ }^{30}$ M. Belloni, W. Christian, and A. J. Cox, Physlet Quantum Physics: An Interactive Introduction (Pearson Prentice Hall, Upper Saddle River, NJ, 2006).

${ }^{31} \mathrm{http}: / /$ web.phys.ksu.edu/vqm

${ }^{32} \mathrm{http}: / /$ www.quantum-physics.polytechnique.fr

${ }^{33}$ http://falstad.com/mathphysics.html

${ }^{34}$ S. B. McKagan, K. K. Perkins, M. Dubson, C. Malley, S. Reid, R. LeMaster, and C. E. Wieman, Developing and researching PhET simulations for teaching quantum mechanics, Am. J. Phys. 76,
406 (2008).

${ }^{35}$ W. K. Adams, S. Reid, R. LeMaster, S. B. McKagan, K. K. Perkins, and C. E. Wieman, A study of educational simulations Part I: engagement and learning, J. Interact. Learn. Res. 19, 397 (2008).

${ }^{36}$ While the transformed curriculum was designed specifically for engineering majors, it was used in the physics majors' course in the semester following this study. This course was not included in the study due to lack of time. Anecdotal observations suggest that the physics majors tended to pick up on things faster, so that the prevalence of many of the difficulties cited here was somewhat less for them, but the qualitative results of this study were not significantly different for this population.

${ }^{37}$ http://per.colorado.edu/QMCS

${ }^{38}$ S. B. McKagan, K. K. Perkins, and C. E. Wieman (unpublished).

${ }^{39}$ A. Elby, What students' learning of representations tells us about constructivism, J. Math. Behav. 19, 481 (2000).

${ }^{40}$ R. W. Gurney and E. U. Condon, Quantum mechanics and radioactive disintegration, Phys. Rev. 33, 127 (1929).

${ }^{41}$ J. B. Pethica and R. Egdell, Surface science: The insulator uncovered, Nature (London) 414, 27 (2001).

${ }^{42}$ D. J. Griffiths, Quantum Mechanics, 2nd ed. (Pearson, Upper Saddle River, 2005).

${ }^{43}$ J. R. Taylor, C. D. Zafiratos, and M. A. Dubson, Modern Physics for Scientists and Engineers, 2nd ed. (Pearson Prentice Hall, Upper Saddle River, 2004).

${ }^{44}$ K. Krane, Modern Physics, 2nd ed. (Wiley, New York, 1996).

${ }^{45}$ R. A. Serway, C. J. Moses, and C. A. Moyer, Modern Physics, 1st ed. (Saunders, Philadelphia, 2005).

${ }^{46}$ H. C. Ohanian, Modern Physics, 2nd ed. (Prentice, Englewood Cliffs, NJ, 1995).

${ }^{47}$ R. Knight, Physics for Scientists and Engineers, 1st ed. (Pearson, San Francisco, 2004).

${ }^{48}$ C. Cohen-Tannoudji, B. Diu, and F. Laloë, Quantum Mechanics (Wiley, New York, 1977).

${ }^{49}$ R. Shankar, Principles of Quantum Mechanics (Springer, New York, 1994).

${ }^{50}$ J. Lande, T. Norsen, and S. B. McKagan, How and why to think about scattering in terms of wave packets instead of plane waves arXiv:0808.3566.

${ }^{51}$ http://phet.colorado.edu/qwi

${ }^{52}$ The Activity-Based Tutorials (Ref. 22), one of which we adapted for use in our curriculum, also include several exercises for building up the idea of potential-energy graphs through laboratory activities with carts on magnetic tracks. We did not use these activities due to lack of time and lack of a laboratory section in our course and because we were more interested in helping students understand potential-energy diagrams for real quantum systems than for classical systems. However, our research indicates that such an approach could be useful if connections are made between these classical examples and examples of systems where quantum mechanics applies. 